\title{
UVB-induced bullous pemphigoid in a patient with psoriasis
}

\author{
Justyna Ceryn, Małgorzata Skibińska, Paulina Barasińska, Marcin Noweta, Joanna Narbutt, Aleksandra Lesiak
}

Department of Dermatology, Pediatric Dermatology and Oncology Clinic, Medical University of Lodz, Lodz, Poland

Adv Dermatol Allergol 2022; XXXIX (1): 228-230

DOI: https://doi.org/10.5114/ada.2020.97725

Bullous pemphigoid (BP) is an autoimmune subepidermal blistering disease affecting mostly elderly people. The clinical picture includes erythematous oedema, excoriated plaques and itchy tense blisters [1]. Drugs, infectious diseases, UV and X-ray radiation are described as some of the triggering factors in the development of the disease [2]. The diagnosis of BP is based on direct immunofluorescence examination (DIF). Typical clinical features, histopathological examination and positive indirect immunofluorescence (IIF) test may be helpful in confirming $\mathrm{BP}$ diagnosis. Coexistence of BP with psoriasis was first reported in 1929 by Bloom [3] and has been investigated since then. Several case reports, including phototherapy as a possible triggering factor were published [4-6].

We present the case of a 71-year-old man with a 4 days' history of tense blisters and erosions on the skin associated with severe itching. Ten days before the appearance of blisters, the patient had completed a 2-month phototherapy course with narrow-band UVB (NB-UVB) started due to an exacerbation of psoriasis. On examination there were numerous tense blisters, erosions and erythematous plaques presented mainly on the upper and lower limbs with no mucous membrane involvement (Figure 1). The examination of other organs and systems was unremarkable. Routine laboratory tests and a chest X-ray showed no abnormalities. DIF of the perilesional skin revealed linear deposits of IgG and C3 at the basement membrane zone and confirmed the diagnosis of BP. The diagnosis was further supported by positive IIF, split-skin biopsy and histopathology.

The patient was started on methotrexate, initially at a dose of $5 \mathrm{mg}$, gradually increased to $10 \mathrm{mg}$ per week I.M. and topical clobetasol propionate $0.025 \%$ cream. The patient tolerated the treatment well and his control blood tests were within normal limits. Over the next 3 months his skin lesions gradually improved until they completely resolved. During the 13-month follow-up appointment only hyperpigmentation on the previously involved skin areas was observed.
Coexistence of psoriasis and bullous pemphigoid is rare, but BP is the most frequent bullous disease described in psoriatic patients [7]. Although it is likely that those patients have a genetic predisposition towards autoimmune diseases, the precise mechanism by which triggering factors (e.g. cyclosporine, biological treatment and phototherapy) are responsible for their development remains unknown. It has been suggested that chronic inflammation around the dermoepidermal junction present in psoriasis can trigger exposure of basement membrane antigens to autoreactive T cells, resulting in BP development [7]. Ho et al. [8] in the nationwide, populationbased cohort study conducted in Taiwan in 2019 proved that psoriasis was independently associated with a 3.05fold increased risk of BP. The research of Phan et al. [7] conducted in 2018 on 4035 bullous pemphigoid patients showed a significantly higher prevalence of psoriasis in BP patients compared to controls (2.6\% vs. $1.1 \%, \mathrm{OR}=$ 2.5, 95\% Cl: 1.4-4.6).

It is interesting that although phototherapy is regarded as one of the most frequent triggering factors in BP development, there are only a handful of articles about PUVA therapy and even fewer about NB-UVB therapy, mainly reported in late 1970s. The mechanism of NB-UVB therapy in the development of BP remains unknown. It is suggested that UV radiation may induce the alteration of basement membrane antigenicity by direct epidermal damage and subsequent autoantibody production, which may later cross-react with basement membrane proteins, such as the BP antigen, causing a bullous eruption [9].

We compared our patient with two, recently published case reports by Suwarsa et al. [9] and Corey et al. [10] of BP development after a course of UVB-NB in psoriatic patients (Table 1). Firstly, in all 3 cases, blisters had appeared after several weeks of starting phototherapy (from 10 to 20 weeks), which seems to question the possibility of NB-UVB therapy being the cause of BP development. Our patient and the patient described by Suwarsa et al. [9] had the diagnosis of BP confirmed

Address for correspondence: Justyna Ceryn MD, Department of Dermatology, Pediatric Dermatology and Oncology Clinic, Medical University of Lodz, Lodz, Poland, e-mail: justyna.ceryn@gmail.com Received: 18.06.2020, accepted: 2.07.2020. 

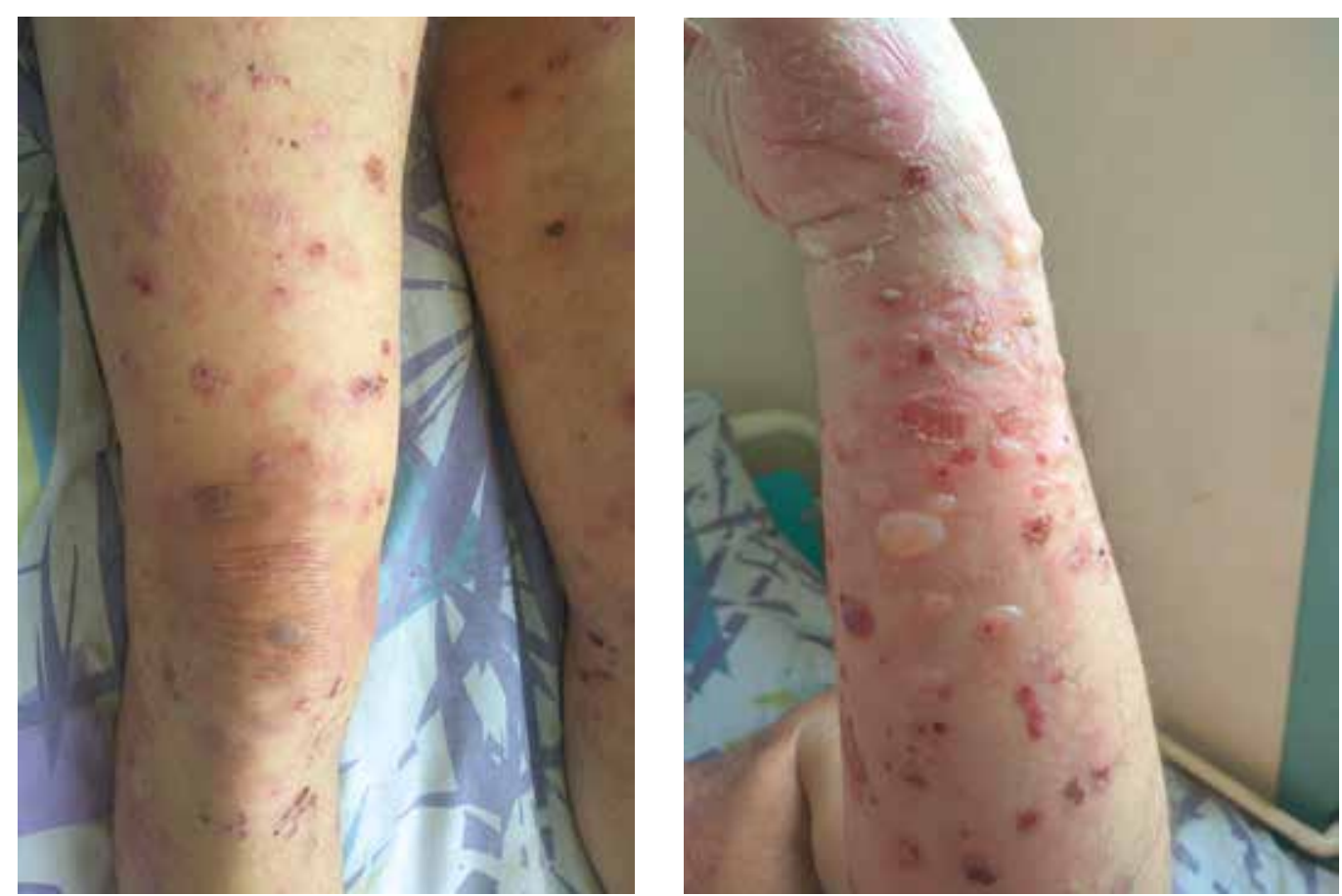

Figure 1. The patient with psoriasis and bullous pemphigoid triggered by NB-UVB treatment

Table 1. Comparison of patients' case reports with BP triggered by NB-UVB phototherapy

\begin{tabular}{|c|c|c|c|}
\hline Patients' details & Suwarsa et al. & Corey et al. & Our patient \\
\hline Age & 39 years old & 49 years old & 71 years old \\
\hline Sex & Male & Male & Male \\
\hline History of psoriasis & 5 years & 4 months & 15 years \\
\hline $\begin{array}{l}\text { Time from starting the NB-UVB } \\
\text { phototherapy to development } \\
\text { of blisters }\end{array}$ & 20 weeks & 11 weeks & 10 weeks \\
\hline PB/BP confirmation & $\begin{array}{c}\text { DIF: linear deposit of IgG and } \\
\text { C3 at the basement membrane } \\
\text { zone }\end{array}$ & $\begin{array}{l}\text { DIF: faint granular C3 } \\
\text { deposition along the } \\
\text { basement membrane } \\
\text { zone }\end{array}$ & $\begin{array}{l}\text { DIF: linear deposit of IgG and C3 in } \\
\text { the dermo-epidermal junction } \\
\text { IIF: circulating pemphigoid-type } \\
\text { antibodies }\end{array}$ \\
\hline Systemic treatment & $\begin{array}{l}\text { Methotrexate }-15 \text { mg/week p.o. } \\
\text { Prednisone }-1 \mathrm{mg} / \mathrm{kg} / \text { day p.o. }\end{array}$ & Topical treatment only & $\begin{array}{l}\text { Methotrexate - initially } 5 \mathrm{mg} / \text { week, } \\
\text { increased to } 7.5 \mathrm{mg} / \text { week) i.m. }\end{array}$ \\
\hline
\end{tabular}

based on DIF. In contrast, the patient described by Corey et al. [10] had questionable DIF with faint granular C3 deposition along the BMZ only suggesting the diagnosis of BP. Only our patient had confirmed IIF with circulating pemphigoid-type antibodies. The results of histopathological examination in all 3 cases suggested BP. Two of the patients had a previous history of psoriasis: of 5 and 15 years, respectively (Suwarsa et al. [9] and our patient), whereas the patient of Corey et al. [10] had a 4 months' history of psoriasis before the development of BP, which would be consistent with published case-control studies confirming that over one third of BP/psoriasis patients are diagnosed with $\mathrm{BP}$ in the first year after psoriasis was confirmed [8]. In our patient and in the one presented by Suwarsa et al. [9] NB-UVB phototherapy was discontinued and the patients were successfully treated with methotrexate (Table 1) and topical corticosteroids. In addition, the patient of Suwarsa et al. [9] was also started on oral prednisone at the dose of $1 \mathrm{mg} / \mathrm{kg} /$ day. The patient described by Corey et al. [10] was treated with topical treatment only. As for the possible diagnosis of $\mathrm{BP}$, it seems controversial that in his case phototherapy was continued within 5 days after the blisters had disappeared, making the diagnosis even less likely.

According to the literature and because some therapies administered for psoriasis can potentially be directly connected to BP development, it is crucial to identify patients in which this scenario is more likely to happen. 
In patients who already have both diagnoses the treatment with oral/intramuscular methotrexate or cyclosporine with additional topical corticosteroids may be advisable [9]. The treatment of BP with systemic corticosteroids needs to be considered with caution as it is not recommended in psoriasis [11].

In conclusion, development of BP in psoriatic patients is rare and can be triggered by some therapies administered for psoriasis. Despite the fact that phototherapy is reported in the literature as one of the most frequent causes of BP in psoriatic patients, further investigations into the mechanisms and occurrence of that presentation need to be performed.

\section{Acknowledgments}

The study was supported by internal funds of the Medical University of Lodz, Lodz, Poland (number: 503/5064-04/503-01).

\section{Conflict of interest}

The authors declare no conflict of interest.

\section{References}

1. Woźniak K, Dmochowski M, Placek W, et al. Pemphigoid - diagnosis and treatment. Polish Dermatological Society Consensus. Dermatol Rev 2016; 103: 19-34.

2. Bağcı IS, Horváth ON, Ruzicka T, at al. Bullous pemphigoid. Autoimmun Rev 2017; 16: 445-55.

3. Bloom D. Psoriasis with superimposed bullous eruption. Med J Rec 1929; 130: 246.

4. Abel EA, Bennett A. Bullous pemphigoid. Occurrence in psoriasis treated with psoralens plus long-wave ultraviolet radiation. Arch Dermatol 1979; 115: 988-9.

5. Washio H, Hara H, Suzuki H, et al. Bullous pemphigoid on psoriasis lesions after UVA radiation. Acta Derm Venereol 2005; 85: 561-3.

6. Barnadas MA, Gilaberte M, Pujol R, et al. Bullous pemphigoid in a patient with psoriasis during the course of PUVA therapy: study by ELISA test. Int I Dermatol 2006; 45: 1089-92.

7. Phan K, Goyal S, Murrell DF. Association between bullous pemphigoid and psoriasis: systematic review and metaanalysis of case-control studies. Australas I Dermatol 2019; 60: 23-8.

8. Ho YH, Hu HY, Chang YT, et al. Psoriasis is associated with increased risk of bullous pemphigoid: a nationwide population-based cohort study in Taiwan. J Dermatol 2019; 46: 604-9.

9. Suwarsa O, Herlina L, Sutedja E, et al. Concurrence of bullous pemphigoid and psoriasis: a case report. Serbian J Dermatol Venereol 2019; 10: 124-8.

10. Corey K, Levin NA, Hure M, et al. Eruption of bullae within psoriatic plaques: a rare adverse effect of narrow-band $u$ traviolet B (NB-UVB) phototherapy. Dermatol Online I 2012; 18: 3.

11. Marek-Jozefowicz L, Scibior K, Czajkowski R. PUVA induced bullous pemphigoid in a patient with psoriasis. Acta Dermatovenerol Croat 2014; 22: 301-4. 